\title{
Embedded P.E.M. Fuel Cell Stack Nonlinear Observer by means of a Takagi-Sugeno Approach
}

\author{
Severus Constantin OLTEANU ${ }^{1}$, Abdel AITOUCHE ${ }^{1}$, Lotfi BELKOURA ${ }^{1}$, Adnan JOUNI ${ }^{\mathbf{2}}$ \\ ${ }^{1}$ Laboratoire CRIStAL (Research Center in Informatics, Signal and Automatic control in Lille), \\ University of Lille 1, \\ Cité Scientifique, Av. Paul Langevin, 59655, Villeneuve D’Ascq Cedex, France \\ severus.olteanu@gmail.com, lotfi.belkoura@univ-lille1.fr, abdel.aitouche@hei.fr \\ ${ }^{2}$ Lebanese University of Beirut, \\ Baabda, Beirut, Lebanon \\ adnan_jouni@yahoo.fr
}

\begin{abstract}
This paper deals with the design of a nonlinear state observer for parameters within the gas transfer part of a fuel cell stack and its implementation on a small-scale embedded system. The fuel cell stack is of a proton exchange membrane type, with parameters specific to vehicle applications. The observer is afterwards applied on a small scale embedded board. In order to validate the embedded observer, a real time hardware in the loop testing is done using a cosimulation between the embedded observer and the professional simulation software AMESim, linked with Simulink on a Windows platform. To act upon the nonlinear character of the system, a Takagi-Sugeno approach is implemented, where the premise variables are unmeasurable. The procedure applies Lyapunov stability theory and by demanding bounded stability instead of asymptotic one, the algorithm manages to eliminate the need for Lipschitz constants.
\end{abstract}

Keywords: nonlinear state estimation, PEM fuel cell, Takagi Sugeno, unmeasurable premise variables, embedded observer, hardware in the loop validation.

\section{Introduction}

The Hydrogen Fuel Cell systems have spurred interest in the last decade, despite the still high production cost, because of their elevated efficiency, reduced pollution level and the targeted independence from fossil fuels. Amongst different types of Fuel Cells (FC) like solid oxide or alkaline ones [1], the proton exchange membrane (P.E.M.) type [2] stands out, because of its low working temperature, and proves to be best suited for vehicle applications. In electrical vehicles, the energy storage plays one of the most important roles [3], so the research on Fuel Cells will boost the acceptance of electrical vehicles as well. Therefore, it directly competes with batteries which have been developing continuously for many years, yet prove inferior in some aspects to hydrogen technology as shown in [4], both as weight per storage capacity and energy density; these represent two important factors, that add to the slow recharge rate of a battery.

The Fuel Cells are small scaled devices therefore the development of virtual sensors would reduce the price. Also, a state observer may be used for diagnostics [5]. The majority of the papers that take into account the dynamics and not only the static models of FCs, focus only upon the electrical part of the fuel cell ignoring the auxiliary components [6] or treating just the compressor separately [7].
Nevertheless, papers such as [8], have to be mentioned as a thorough review upon all the components used so far. Indeed, for the more general case of system diagnosis, we find also many alternative approaches to model based techniques (for which a good review is [9]): experimental (ex: impedance spectroscopy [10], neuro-fuzzy techniques). As there is still no standardization in different existing types of FCs, a functional model would be easier to adapt to any particular case instead of experimental approaches that require extensive training data. Also model based approaches [11], [12] have the potential to give fast response to time variations, therefore being very efficient for on-line diagnosis [13] as well as control [14]. Of course one has to mention the greatest inconvenient of model based techniques that is the difficulty in parameter estimation. The state observer acts as a virtual sensors and it is designed to estimate cathode and anode pressures and mass flows of oxygen and hydrogen which are generally not measured. The mass flow rates of reactant gases play a pivotal role in the reliable and efficient operation of FCS.

For the design of the nonlinear observer, a Takagi-Sugeno (TS) representation has been chosen [15],[16]. This method can be found in literature, acting upon different types of industrial processes [17]. This approach has an advantage over other nonlinear ones in that 
there is no need for many assumptions regarding the form of the state space model, it has a structured form, it is easy to implement numerically and it also allows a parallel to linear techniques to be drawn. The construction of state observers based on TS representation has been in a continuous augmentation in the last period. Although many papers consider the premise variables measurable [19], this case in many practical applications is unfortunately unattainable. Among those who have tackled the issue of unmeasurable premise variables, one can cite [18].

It is useful to adopt the use of simulation software to replace the real system in the first hardware in the loop testing stage. For this, AMESim has been chosen [20]. Also, in the last years, small scaled embedded systems have become more and more accessible.

We can distinguish three classes:

- Microcontroller (based boards) as Arduino boards;

- FPGA which are good for parallel computing;

- Processor based: as Raspberry PI, Beagle board, that act like small computers.

Each of them has certain advantages and disadvantages. In this article the authors have adopted the use of an Arduino Due board, and the development procedure with the hardware in the loop testing being described in [21].

The paper is organized as follows. Second section develops upon the Fuel Cell model. It is followed by a description of the TS representation and nonlinear observer design in the section III. Afterwards, in the fourth section, the embedded platform is described as well as the hardware in the loop (HIL) validation mechanism. The paper ends with results in section $\mathrm{V}$ respectively conclusions in section VI.

\section{Modeling}

\subsection{PEM fuel cell}

The developed model is for a Polymer Electrolyte Membrane Fuel Cell Stack that uses a Nafion 117 membrane, integrating auxiliary components as in Figure 1. The model focuses on components with medium time dynamics from a vehicle like configuration, meaning the focus is on the gaseous part. In vehicle applications, the auxiliary components play an important role, as the piping occupies significant volume.

The current work considers the case of pure Oxygen as input on the cathode side and takes the humidifying and cooling units as ideal elements. Concerning the pressure difference between the anode and cathode, it is kept null by means of a pressure regulator. A constant pressure difference has been seen to offer good performances in many cases.

The temperature inside the fuel cell is considered homogeneous, so spatial variations in general are ignored, yet the temperature is not constant, compared to [22].

Only by measuring parameters outside the fuel cell with the aid of the observer, will allow us to deduce what happens inside the anode and cathode respectively. Moreover, the gases will be considered ideal.

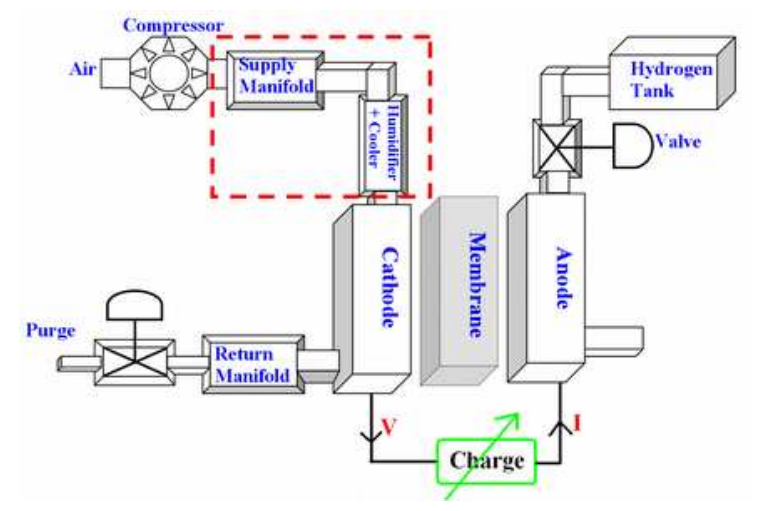

Figure 1. Block view of fuel cell stack with auxiliary elements

\subsection{State Space representation}

The mathematical model of the FC is brought in a state space form considering the compressor's flow and electrical current as inputs to the system. Therefore, the accumulated mass of oxygen and hydrogen react or pass freely towards the return manifold that consists of pipes and valves. The valve models for gaseous mediums have a nonlinear behaviour with two distinct patterns depending on the pressure difference that arises: choked or unchoked regime. Therefore, as the pressure difference may overpass 2 bar both situations have to be taken into account, this being another contribution of the paper. The TS representation will help with this, so that a switching between the two may not be required. The equations for a volume chamber and a valve are given by 


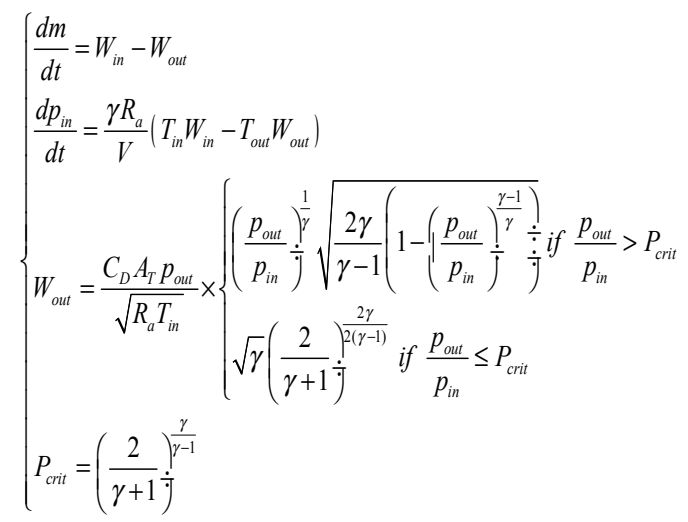

where $m$ is the mass inside the chamber, $p_{i n}, T_{i n}$ and $\mathrm{W}_{\text {in }}$ the input pressure, temperature and flow, $\mathrm{p}_{\text {out }}, \mathrm{T}_{\text {out }}$ and $\mathrm{W}_{\text {out }}$ the output pressure, temperature and flow, $\mathrm{V}$ the volume of the chamber, $\gamma$ the adiabatic coefficient of air, Ra the specific perfect gas constant of air (Oxygen for the current case), $C_{D}$ the flow coefficient of the valve and $\mathrm{A}_{\mathrm{T}}$ the cross section of the valve. The input flow of the supply manifold will be considered as an input to the system with known temperature and pressure and the return manifold will flow toward exterior air.

Regarding the whole FC model, we will suppose that we measure the pressures and mass at the supply and return manifold, as indeed the measurement of the pressure and mass inside the cathode is expensive and impractical. Of course, in order to avoid measuring mass, one can have a pressure and temperature sensor in order to deduce the mass by means of the perfect gas law $P V=m R_{a} T$.

One can represent the equations for the cathode side of the Fuel cell as in (2) (the hydrogen side is identical), defining the dynamics for the mass flows of the Supply Manifold-CathodeReturn manifold, as well as the dynamics of the pressures of the same three elements:

$\left\{\begin{array}{l}\frac{d m_{s m}}{d t}=W_{\text {in }}-W_{\text {outsm }} \\ \frac{d m_{c s}}{d t}=W_{\text {outsm }}-W_{\text {outcs }}-W_{O_{2} \text { react }} \\ \frac{d m_{r m}}{d t}=W_{\text {outcs }}-W_{O_{2} \text { react }}-W_{\text {outrm }} \\ \frac{d p_{s m}}{d t}=\frac{\gamma R_{a}}{V_{s m}}\left(T_{\text {smin }} W_{\text {in }}-T_{\text {sm }} W_{\text {outsm }}\right) \\ \frac{d p_{c s}}{d t}=\frac{\gamma R_{a}}{V_{c s}}\left(T_{c s i n} W_{\text {outsm }}-T_{c s} W_{\text {outcs }}-T_{c s} W_{O_{2} \text { react }}\right) \\ \frac{d p_{r m}}{d t}=\frac{\gamma R_{a}}{V_{r m}}\left(T_{r m i n} W_{\text {outcs }}-T_{r \text { min }} W_{O_{2} \text { react }}-T_{r m} W_{\text {outrm }}\right)\end{array}\right.$
Where the mass flow terms in (2) that represent the mass flow output of the Supply manifold, Return manifold, Cathode and the Mass flow that reacts in the cell are shown in (3). We can see the influence of the electrical current I, on the reacted mass flow depending on the number of cells(n), Oxygen molar mass and Faraday number.

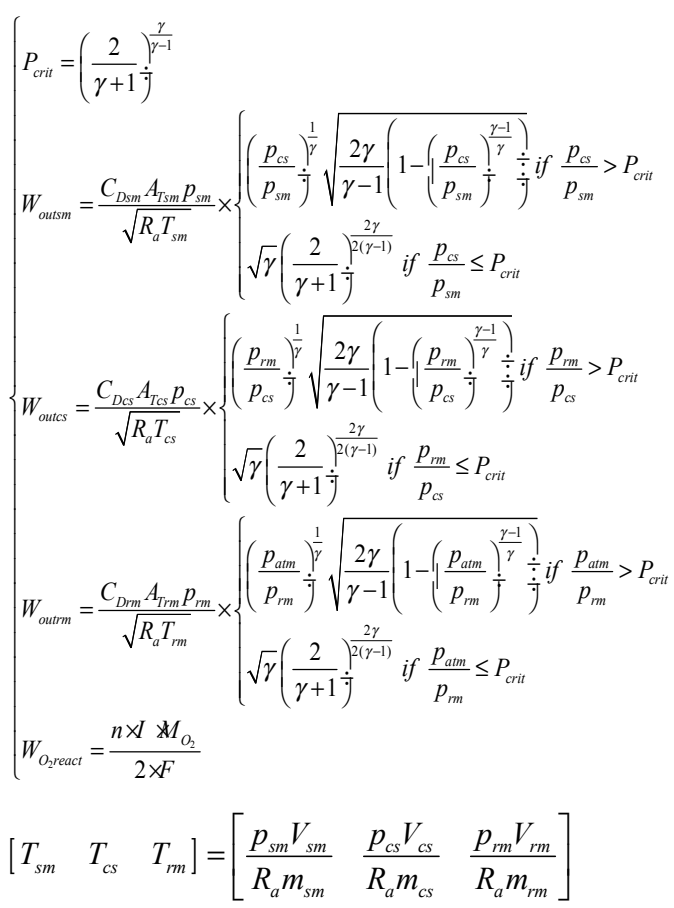

Therefore, by doing a set of transformations and by considering the current as a system input along with the mass flow of the compressor we arrive at its state space equivalent in (4). The critical pressure is the same as the one presented in equation (1).

$$
\begin{aligned}
& u=\left[\begin{array}{ll}
W_{i n} & I
\end{array}\right]^{T} \\
& x=\left[\begin{array}{llllll}
m_{s m} & m_{c s} & m_{r m} & p_{s m} & p_{c s} & p_{r m}
\end{array}\right]^{T} \\
& \dot{x}=A(x)+B u \\
& y=\left[\begin{array}{l}
m_{s m} \\
m_{r m} \\
p_{s m} \\
p_{r m}
\end{array}\right]=\left[\begin{array}{llllll}
1 & 0 & 0 & 0 & 0 & 0 \\
0 & 0 & 1 & 0 & 0 & 0 \\
0 & 0 & 0 & 1 & 0 & 0 \\
0 & 0 & 0 & 0 & 0 & 1
\end{array}\right] x
\end{aligned}
$$

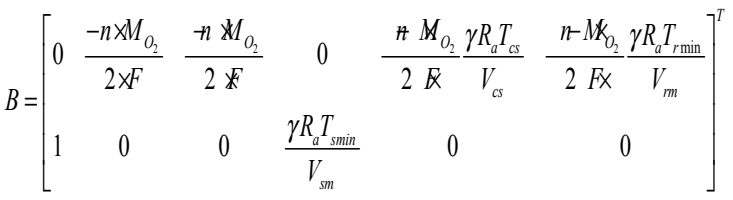

The matrix A being the carrier of the nonlinear terms. 


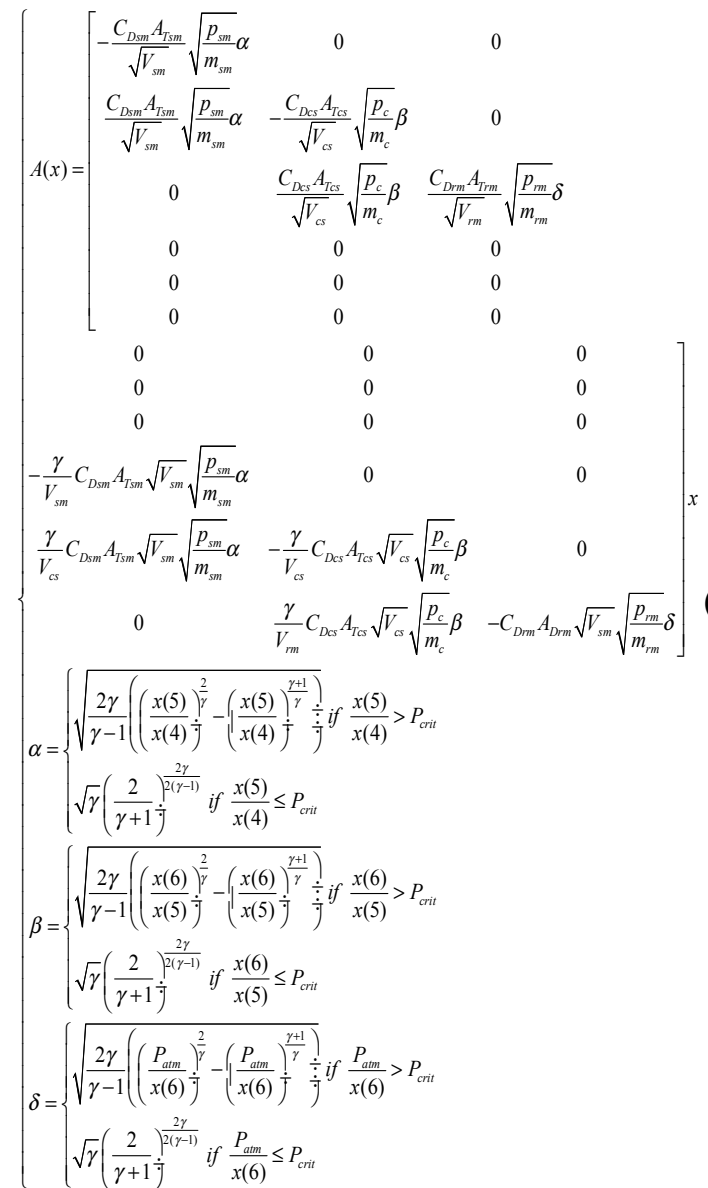

For the values, please refer to the annex.

\section{Observer Design}

\subsection{Takagi-Sugeno representation}

The TS model is represented as a sum of nonlinear systems, where the contribution of each system is corrected by a weighing function $\mathrm{w}_{\mathrm{i}}$, as presented in equation (7). Here, it is considered that $\mathrm{B}$ and $\mathrm{C}$ do not depend on the states and the notation ' $\mathrm{i}$ ' is used to show the number of the decomposed linear system; also $\mathrm{z}$ represents the nonlinear terms, defined as premise variables.

$\left\{\begin{array}{l}\dot{x}=\sum_{i=1}^{8} w_{i}(z) A_{i} x+B u \\ y=C x\end{array}\right.$

Therefore, in this new representation, one can observe that the nonlinearities have moved into the membership functions $\mathrm{w}_{\mathrm{i}}$, where the convex sum property is satisfied $\sum \mathrm{w}_{\mathrm{i}}=1$.

In order to obtain the state observer, it is first needed to modify the state space form, so that it can be used to write the Takagi-Sugeno (TS) representation. So from equation (4), in order to get to the form (7) we separate in the matrix A the $\mathrm{z}$ nonlinearities:

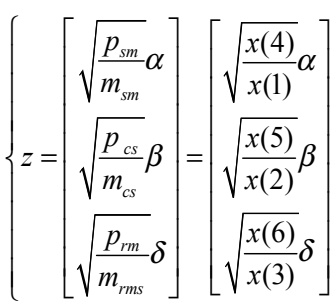

The variables $\mathrm{z}$ are called premise variables, and they contain the nonlinear information for the system. By means of the sector nonlinearity method, as the minimum and maximum of each $\mathrm{Z}_{\mathrm{i}}$ can be determined, the membership functions can be built.

Table 1. Min Max values

\begin{tabular}{|c|c|}
\hline Symbol & Quantity \\
\hline$z_{1_{\text {min }}}=1$ & $z_{1_{\text {max }}}=1500$ \\
\hline$z_{2_{\text {min }}}=3$ & $z_{2_{\text {max }}}=7000$ \\
\hline$z_{3_{\text {min }}}=3$ & $z_{3_{\text {max }}}=1500$ \\
\hline
\end{tabular}

From different types of possible membership functions, triangular membership function were chosen as in (9); where MF is the membership function associated to the $i$-th premise variable $\mathrm{Z}_{\mathrm{i}}$. With these values, one is able to construct the normalized membership functions $\mathrm{w}_{\mathrm{i}}$ as in (10).

$M F_{\text {min }}\left(z_{i}\right)=\frac{z_{i, \text { Max }}-z_{i}}{z_{i, \text { Max }}-z_{i, \text { min }}} ; M F_{\text {Max }}\left(z_{i}\right)=\frac{z_{i}-z_{i, \text { min }}}{z_{i, \text { Max }}-z_{i, \text { min }}}$

$\left\{\begin{array}{l}i=1 . .8 \\ h_{i}=M F_{\text {Max/Min }}\left(z_{1}\right) \times M F_{\text {Max } / M i n}\left(z_{2}\right) M F_{\text {Max } / M i n}\left(z_{3}\right) \\ -------------------- \\ w_{i}=\frac{h_{i}}{\sum_{i=1}^{2^{3}} h_{i}} ;\end{array}\right.$

\subsection{Takagi-Sugeno observer}

Once the T-S representation has been obtained, the focus can now be directed towards the observer construction. The observer computation will materialize as an optimization problem that will deal with the resolution of some linear matrix inequalities (LMIs). It is considered that the premise variables $z$ are not measurable. Although many cases in the literature deal with the simplifying supposition of measurable premise variables $\mathrm{w}_{\mathrm{i}}(\hat{\mathrm{z}})=\mathrm{w}_{\mathrm{i}}(\mathrm{z})$, in the current case this assumption cannot be satisfied. As a result, the Luenberger like observer is of the form (11). 


$$
\left\{\begin{array}{l}
\dot{\hat{x}}=\sum_{i} w_{i}(\hat{z})\left(A_{i} \cdot \hat{x}+B \cdot u+L_{i} \cdot(\hat{y}-y)\right) \\
\hat{y}=C \cdot \hat{x}
\end{array}\right.
$$

To obtain the observer gains, a numerical optimization problem (12) should be resolved, with the aid of an LMI solver. This solution consists of the following LMIs (12); where $\mathrm{P}$ is a symmetric and positive definite matrix, with the same dimension as $\mathrm{A}$; and $\alpha$ is a positive scalar:

$$
\left\{\begin{array}{l}
i=1 . .8 \\
\left(\begin{array}{ll}
A_{i}^{T} P-C^{T} Q_{i}+P A_{i}-Q_{i} C+2 \alpha P & P \\
P & -\lambda \dot{j}
\end{array}\right) \\
Q_{i}=P \times L_{i} \\
\operatorname{size}\left(Q_{i}\right)=\operatorname{size}\left(A_{i} \cdot C\right)
\end{array}\right.
$$

\subsection{Demonstration}

In the demonstration that follows, the known inequality (13) is employed:

$$
\forall \lambda>0, X, Y: X^{T} Y+Y^{T} X \leq \lambda X^{T} X+\lambda^{-1} Y^{T} Y
$$

The condition required for the observer to function correctly is to have an estimation error that converges towards zero $\tilde{x}=x-\hat{x}$.

Using the Lyapunov stability method, it is demanded that a chosen Lyapunov function has a negative derivative. The Lyapunov function is chosen as in (14).

$$
V=\widetilde{x}^{T} P \widetilde{x} ; P=P^{T}>0
$$

The derivative of the estimation error can be rewritten so that the estimated and real membership functions multiplied by the states are isolated in a separate term. This is done in order to reduce complications that would appear in the construction of the LMIs. Therefore (15) is reached.

$$
\left\{\begin{array}{l}
\dot{\tilde{x}}=\sum_{i} w_{i}(\hat{z})\left(A_{i} \cdot \hat{x}+B \cdot u+L_{i} \cdot C \cdot \tilde{x}\right)+\Delta ; \\
\Delta \stackrel{n o t}{=} \sum_{i}\left(w_{i}(z)-w_{i}(\hat{z})\right)\left(A_{i} \cdot x+B \cdot u\right)
\end{array}\right.
$$

By replacing (15) into (14), we can see in (16) that the multiplication with the state impedes obtaining a linear inequality. A usual workaround is to apply Lipschitz constants, but the method could prove restrictive, first of all because these values are hard to find and also high constants may have negative effects on the resolution of the LMIs.

$$
\dot{V}=\tilde{x}^{T}\left(\begin{array}{c}
\sum_{i} w_{i}(\hat{z})\left(A_{i}-L_{i} C\right)^{T} P+ \\
+\sum_{i} w_{i}(\hat{z}) P\left(A_{i}-L_{i} C\right) \\
+\Delta^{T} P \tilde{x}+\tilde{x}^{T} P \Delta
\end{array}\right) \tilde{x}+
$$

As such, the solution adopted here is to view $\Delta$ as a virtual perturbation, a parameter that converges towards zero. This would allow us to impose more relaxed conditions, by demanding a bounded stability instead of an asymptotic one, with a minimal bound. An article with a similar method is [18], employing the notion of Input to state stability [24]. Practically this translates into a problematic of stability with rejection of the symbolic perturbation $\Delta$, as in (17), having $\mathrm{R}$ as a positive matrix (identity or not), and a positive scalar $\xi$ representing a variable to be minimized.

$$
\dot{V}+\tilde{x}^{T} R \tilde{x}-\xi^{2} \Delta^{T} \Delta<0
$$

Therefore, using (16):

$$
\begin{aligned}
& \tilde{x}^{T}\left(\sum_{i} w_{i}(\hat{z})\left[\left(A_{i}-L_{i} C\right)^{T} P+P\left(A_{i}-L_{i} C\right)\right] \mid \tilde{x}+\right. \\
& +\Delta^{T} P \tilde{x}+\tilde{x}^{T} P \Delta+\tilde{x}^{T} R \tilde{x}-\xi^{2} \Delta^{T} \Delta<0
\end{aligned}
$$

In order to eliminate the nonlinear terms that remain in the previous inequality, we make use of the equation (13). What is important to note is that this is true for any positive $\lambda$, which for (18) translates into:

$$
\begin{aligned}
& \Delta^{T} P \tilde{x}+\tilde{x}^{T} R \tilde{x}-\xi^{2} \Delta^{T} \leqslant \\
& \lambda \Delta^{T} \Delta+\lambda^{-1} \tilde{x}^{T} P P \tilde{x}+\tilde{x}^{T} R \tilde{x}-\xi^{2} \Delta^{T} \Delta
\end{aligned}
$$

Adding this to (18) would give the modified inequality (20):

$$
\begin{aligned}
& e^{T}\left(\sum_{i} w_{i}(\hat{z})\left[\left(A_{i}-L_{i} C\right)^{T} P+P\left(A_{i}-L_{i} C\right)\right]+I_{\dot{j}}\right) e+ \\
& +\lambda \Delta^{T} \Delta+\lambda^{-1} e^{T} P P e-\xi^{2} \Delta^{T} \Delta<0 ;
\end{aligned}
$$

An interesting observation is that, because $\lambda>0$ can be any coefficient, then it can be chosen as $\lambda=\xi^{2}$, which simplifies the relation; moreover, employing the convex sum property, we arrive at (21). A similar idea was used in [23].

$$
\sum_{i} w_{i}(\hat{z})\left[\left(A_{i}-L_{i} C\right)^{T} P+P\left(A_{i}-L_{i} C\right)+I+\lambda^{-1} P P\right]<0 ;
$$

The general following stage is to use the hypothesis that the inequality is true if all the terms of the sum are negative. Although restrictive, this proves to bring small enough constraints to the stability regions of the solution. 
$\left\{\begin{array}{l}i=1 . .8 \\ \left(A_{i}-L_{i} C\right)^{T} P+P\left(A_{i}-L_{i} C\right)+I+\lambda^{-1} P P<0 ;\end{array}\right.$

In order to eliminate the Bilinear Matrix Inequalities (BMIs) forms, a notation $Q_{i}=P L_{i}$ can be made. Also using the Schur transformation, we end up with simple LMIs. One can improve upon the performances by asking for exponential stability, using relation (23), where $\alpha$ represents the exponential decay rate.

$$
\dot{V}=e^{T} R e-\xi^{2} \Delta^{T} \Delta+2 \alpha V<0
$$

As for BMIs there are no standard solutions, a choice of $\alpha$ by trial can be made.

Thus LMIs (12) were obtained.

\section{The Embedded Solution}

\subsection{The Arduino board}

The embedded platform chosen is an Arduino Due board with a 32 bit ARM microcontroller from Atmel with 84Mhz clock speed (Figure 3).

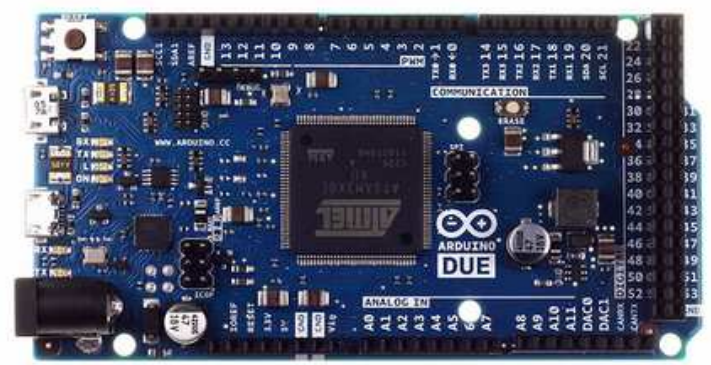

Figure 2. The Arduino DUE microcontroller based board

The power of the microcontroller comes from the fact that it is based on an ARM Cortex M3 processor (Atmel SAM3x8E). An Ethernet module is attached for data communication.

\subsection{Hardware in the loop testing}

The integration of specialized simulation software can be done by means of hardware in the loop (HIL) architecture which permits within a closed loop with a physical embedded system, to test the code and performance of the developed platform. By means of AMESim simulation software, that includes a Fuel Cell model amongst other components, a real time connection can be established between the embedded system and the computer. For the computer, a Windows operating system has been chosen, because although it is not well suited for real time operation is the most widespread and known one in the academic medium.

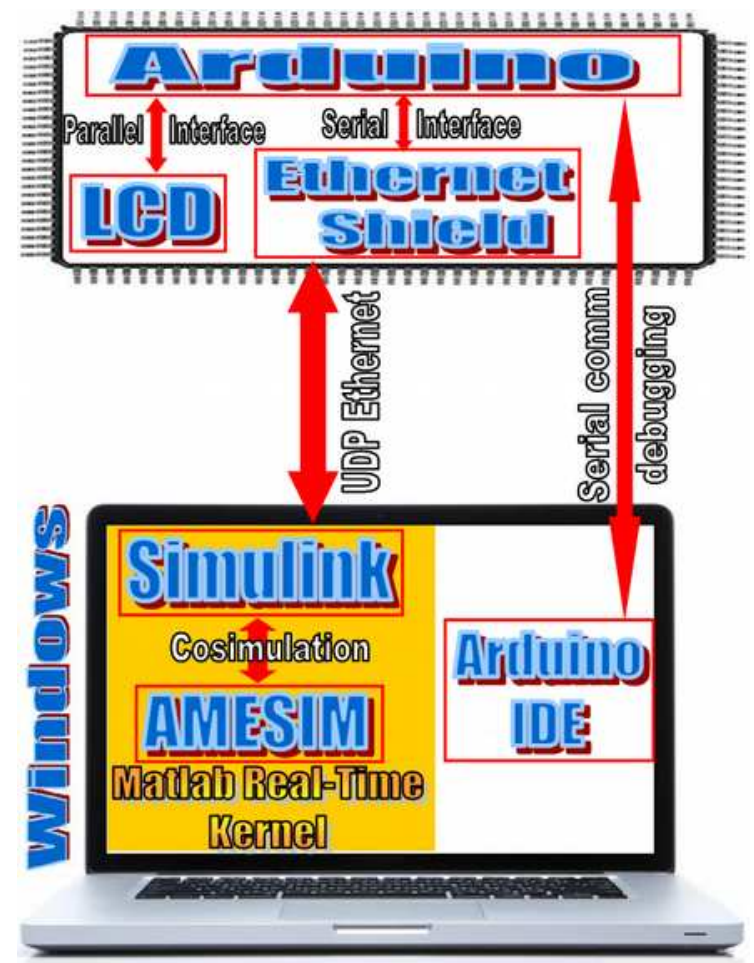

Figure 3. The embedded HIL validation schematics

In order to give real time capabilities to windows, Matlab Real Time Kernel and Simulink are used as an interface. A functional schematic is shown in Figure 4. The tested communication protocols were serial and Ethernet, offering an as general solution as possible. For more details see [21]. The AMESim Fuel cell model built is presented in Figure 5.

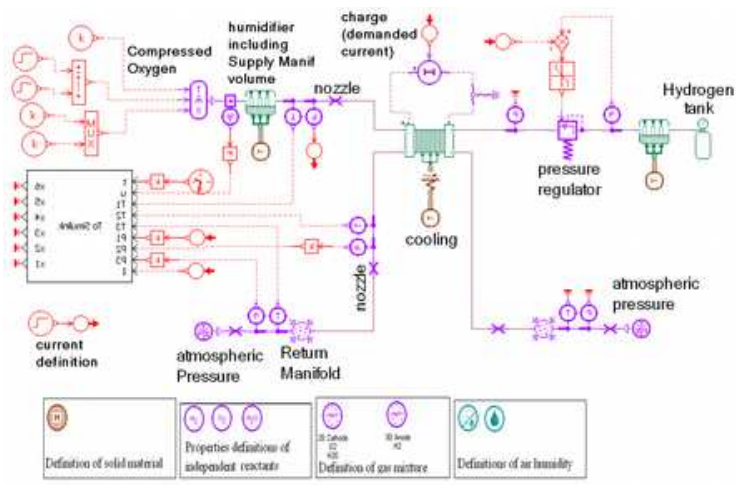

Figure 4. The Amesim model of the PEM FC

\section{Implementing the Embedded Observer}

Using the Matlab LMI solver for the previous LMIs applied to the system at hand, we obtain the observer gains which are implemented on the Arduino board, by means of discretization. At this point in order to check the performance we will use different initial values for the observer states. 
$\hat{x}(0)=\left[\begin{array}{llllll}0.0563 & 0.0019 & 0.0380 & 387419.1 & 170226 & 144894.8\end{array}\right]^{T}$

The simulation between AMESim, Simulink and the Arduino in real time gives us the response in the following figures.

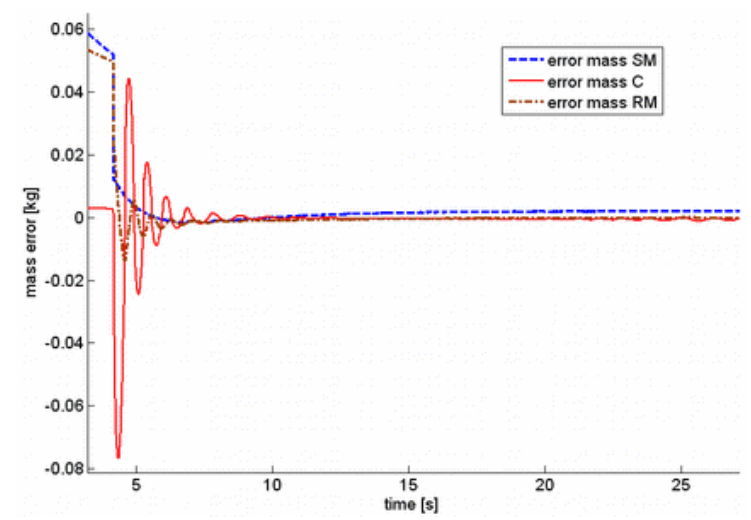

Figure 5. Unmeasured premise variables observer error (difference between real and estimated value) for the gas masses of the oxygen supply of the PEMFC

The observer appears inactive in the first few seconds due to the initialization time of the devices and the communication.

By following the evolution of the estimation error in Figure 7, one can notice that the stability is not asymptotic, but bounded, of course the bounds being sufficient to respond to requirements. Also one can notice that indeed in the beginning, the observer manifests oscillations before stabilizing, but the settling time is still in an acceptable interval.

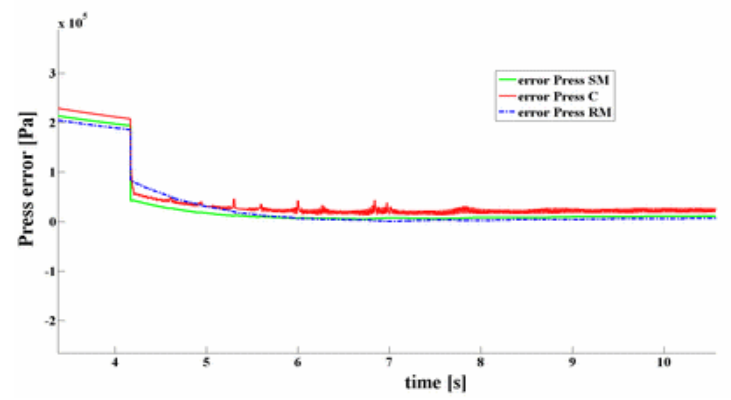

Figure 6. Pressure estimation error of the oxygen supply of the PEMFC

One can observe in Figure 6, at a time around 7 seconds for example, that there is a communication loss, yet the observer manages to readapt the estimated values to the real ones. The few seconds delay at the beginning is just due to the initialization procedure of the embedded system.

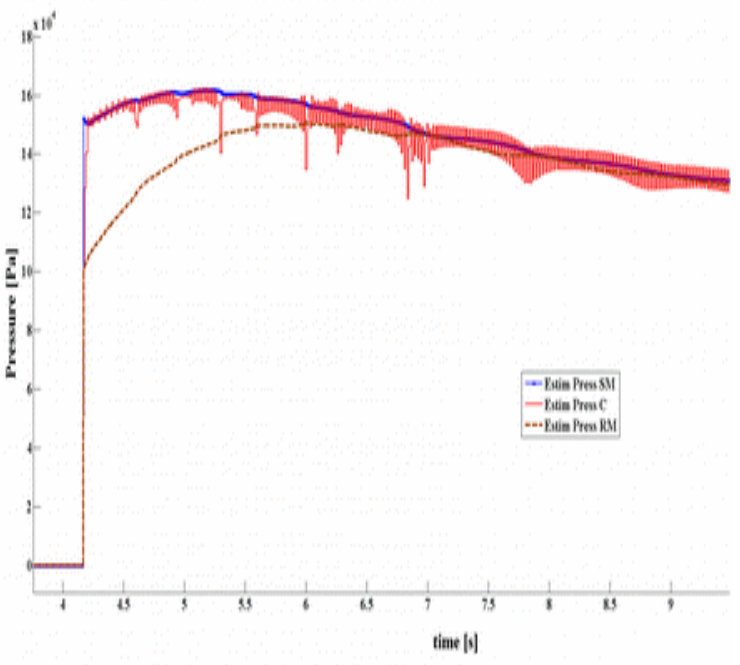

Figure 7. Real time observer values for the pressures of the oxygen supply of the PEMFC

The main problem with this particular embedded system is that the dynamic of the valve is fast and that, for a sample time of more than $5 \mathrm{~ms}$, oscillations appear. Considering that we have $6 \times 6$ and $6 \times 4$ matrices, an 8 bit microcontroller arrives at a minimum sampling time of $10-15 \mathrm{~ms}$. Therefore we need to use the Arduino DUE, a 32 bit processor, which has $96 \mathrm{kBytes}$ of RAM. Furthermore, the Arduino DUE has a $84 \mathrm{MHz}$ clock, allowing to do the floating point calculations for the oxygen supply in $4 \mathrm{~ms}$.

\section{Conclusions}

In this paper, we have developed a TS unmeasurable premise variable observer for determining parameters inside a PEM Fuel Cell which is able to estimate the pressures and mass flow rates. The paper also shows the potential of Takagi-Sugeno modeling to be applied on small scale processing units, achieving good sampling time and real-time constraints. Thus, such solution can be embedded on an electrical vehicle supplied by a FC system, being more economically viable than the usual ECU. The real-time interface between AMESim, Matlab/Simulink and an Arduino connected by Ethernet that we developed represents a proper way to test any FC related embedded system and ensures that the observers behave as intended before using them on a real system. Using this connection allows to test any possible loss in the communication and design of the observer. Despite the memory restriction, the calculations remain fast and the limiting factor for real-time 
can be improved by parallel calculus as T-S is well adapted for it.

\section{Acknowledgments}

This work was carried out in the framework of PN-7-022-BE i-MOCCA (Interregional Mobility and Competence Centres in Automation), an European territorial cooperation project part-funded by the European Regional Development Fund (ERDF) through the INTERREG IV A 2 Seas Programme and the Ministry of Education and Research, France.

\section{REFERENCES}

1. KIRUBAKARAN, A., S. JAIN, R. K. NEMA, A Review on Fuel Cell Technologies and Power Electronic Interface, Renewable and Sustainable Energy Reviews, vol. 13, no. 9, December 2009, pp. 2430-2440.

2. BARBIR, F., PEM Fuel Cells Theory and Practice. Second edition, Ed. Elsevier Inc., 2013.

3. LUKIC, S. M., J. CAO, R. C. BANSAL, F. RODRIGUEZ, A. EMADI, Energy Storage Systems for Automotive Applications, IEEE Transactions on Industrial Electronics, vol. 55, no. 6, June 2008, pp. 2258-2267.

4. THOMAS, C. E., Fuel Cell and Battery Electric Vehicles Compared, International Journal of Hydrogen Energy, vol. 34, Jan 2009, pp. 6005-6020.

5. ZHANG, J., H. ZHANG, J. WU, J. ZHANG, Techniques for PEM Fuel Cell Testing and Diagnosis, PEM Fuel Cell Testing and Diagnosis, Elsevier, 2011, pp. 81-119.

6. KIM, J., J. LEE, B. H. CHO, Equivalent Circuit Modeling of PEM Fuel Cell Degradation Combined with a LFRC, IEEE Transactions on Industrial Electronics, vol. 60, no. 11, November 2013, pp. 5075-5085.

7. MATRAJI, I., S. LAGHROUCHE, M. WACK, Cascade Control of the MotoCompressor of a PEM Fuel Cell via Second Order Sliding Mode, Decision and Control and European Control
Conference (CDC-ECC), 2011 50th IEEE Conference, December 2011, pp.633-638.

8. PUKRUSHPAN, J. T., A. G. STEFANOPOUlOU, H. PENG, Control of Fuel Cell Power Systems: Principles, Modeling, Analysis and Feedback Design. Ed. Springer, 2004.

9. PETRONE, R., Z. ZHENG, D. HISSEL, $M$. C. PERA, C. PIANESE, M. SORRENTINO, M. BECHERIF, N. Y. STEINER, A Review on Model-based Diagnosis Methodologies for PEMFCs, International Journal of Hydrogen Energy, vol. 38, no. 17, 2013, pp. 7077-7091.

10. STEINER, N. Y., D. HISSEL, P. MOÇOTÉGUY, D. CANDUSSO, Non Intrusive Diagnosis of Polymer Electrolyte Fuel Cells by Wavelet Packet Transform, International Journal of Hydrogen Energy, vol. 36, no. 1, 2011, pp. 740-746.

11. HOSSEINI, M., A. H. SHAMEKHI, A. YAZDANI, Modeling and Simulation of a PEM Fuel Cell (PEMFC) Used in Vehicles, SAE 2012 World Congress \& Exhibition 2012, 2012-01-1233.

12. AITOUCHE, A., Q. YANG, B. OULD BOUAMAMA, Fault Detection and Isolation of PEM Fuel Cell System based on Nonlinear Analytic Redundancy. An Application Via Parity Space Approach, The European Physical Journal of Applied Physics, vol. 54, January 2011.

13. HAFAIFA, A., F. LAAOUAD, K. LAROUSSI, Fuzzy Approach Applied in Fault Detection and Isolation to the Compression System Control, Studies in Informatics and Control, ISSN 1220-1766, vol. 19, no. 1, 2010, pp. 17-26.

14. KIM, E. S., Observer Based Nonlinear State Feedback Control of PEM Fuel Cell Systems, Journal of Electrical Engineering and Electronic Technology vol. 7, no. 6, 2012, pp. 891-897.

15. TANAKA, K., T. IKEDA, H. WANG, Fuzzy Regulators and Fuzzy Observers: Relaxed Stability Conditions and LMIbased Designs, IEEE Transactions on Fuzzy Systems, vol. 6, no. 2, 1998, pp. 250-265.

16. JAMEL, W., A. KHEDHER, N. BOUGUILA, K. B. OTHMAN, State 
Estimation via Observers with Unknown Inputs: Application to a Particular Class of Uncertain TakagiSugeno Systems, Studies in Informatics and Control, ISSN 1220-1766, vol. 19, no. 3, 2010, pp. 219-228.

17. GEORG, S., M. MULLER, H. SCHULTE, Wind Turbine Model and Observer in Takagi-Sugeno Model Structure, Journal of Physics: Conference Series, vol. 555, no $1,2014$.

18. YACINE, Z., D. ICHALAL, N. AIT OUFROUKH, S. DJENNOUNE, Nonlinear Vehicle Lateral Dynamics Estimation with Unmeasurable Premise Variable Takagi-Sugeno Approach, $20^{\text {th }}$ Mediterranean Conference on Control \& Automation (MED), Barcelona, Spain, July 2012, pp. 1117-1122.

19. LENDEK, Z., T. M. GUERRA, R. BABUSKA, B. de SCHUTTER, Stability Analysis and Nonlinear Observer Design Using Takagi-Sugeno Fuzzy Models, Studies in Fuzziness and Soft Computing, Ed. Springer, 2010.

20. BOURDON, T., L. SAUSSOL, B. VAROQUIÉ, Integration of Physical AMESim ${ }^{\circledR}$ Engine Model in Hardware in the Loop Environment, Dedicated to Engine Control Unit Testing, SAE Technical Paper, January 2007.
21. OlTEANU, S. C., A. AitOUCHE, L. BELKOURA, Advanced Embedded Nonlinear Observer Design and HIL Validation Using a Takagi-Sugeno Approach with Unmeasurable Premise Variables, Journal of Physics: Conference Series, vol 570, no. 2, 2014.

22. OlteANU, S. C., A. AITOUCHE, L. BELKOURA, Advances in P.E.M. Fuel Cell Stack Observer Design Using Takagi-Sugeno Approach with Unmeasurable Premise Variables, ICREGA 2014 Renewable Energy: Generation and Applications, Springer Proceedings in Energy, Chapter 10, 2014, pp. 117-130.

23. GHORBEL, H., A. El HAJJAJI, M. SOUISSI, M. CHAABANE, Robust Tracking Control for Takagi-Sugeno Fuzzy Systems With Unmeasurable Premise Variables: Application to Tank System, Journal of Dynamic Systems, Measurement, and Control, vol. 136, no. 4, 2014.

24. SONTAG, E. D., On the Input-to-State Stability Property, Journal Systems \& Control Letters, vol. 24, 1995, pp. 351-359. 


\section{Annex}

Table 2. Parameter Values

\begin{tabular}{|c|c|c|c|}
\hline Symbol & Value & Symbol & Value \\
\hline$\gamma$ & 1.4 & $A_{T c}$ & $600 \mathrm{~mm}^{2}$ \\
\hline$R_{a}$ & $259.8 \frac{J}{k g . K}$ & $A_{T r m}$ & $600 \mathrm{~mm}^{2}$ \\
\hline$C_{D s m}$ & 0.2 & $V_{s m}$ & $20 \mathrm{~L}$ \\
\hline$A_{T s m}$ & $550 \mathrm{~mm}^{2}$ & $V_{c}$ & $1 \mathrm{~L}$ \\
\hline$V_{s m}$ & $20 \mathrm{~L}$ & $V_{r m}$ & $20 \mathrm{~L}$ \\
\hline$C_{D s m}$ & 0.2 & $T_{s m_{\text {in }}}$ & $353.15 \mathrm{~K}$ \\
\hline$C_{D c}$ & 0.2 & $P_{s m_{\text {in }}}$ & $5 \mathrm{bar}$ \\
\hline$C_{D r m}$ & 0.075 & $T_{r m_{o u t}}$ & $273.15 \mathrm{~K}$ \\
\hline$A_{T s m}$ & $550 \mathrm{~mm}^{2}$ & $P_{s m_{\text {out }}}$ & $1.013 \mathrm{bar}$ \\
\hline
\end{tabular}

